\title{
O DESIGN PARA ADAPTABILIDADE (DFAD) NA ARQUITETURA CONTEMPORÂNEA BRASILEIRA: CONSIDERAÇÕES A PARTIR DE UMA REVISÃO SISTEMÁTICA DA LITERATURA
}

José Evandro de Moura Rosa Henriques/UFPE e Loughborough University Amilton Arruda/UFPE

Robert Schmidt III/Loughborough University

\section{RESUMO}

O Design para Adaptabilidade ( $D f A D)$ tem se revelado um tópico de interesse mundial acerca do futuro da Arquitetura e à problemática da obsolescência nas edificações. Em países como o Brasil, onde existem altos índices de geração de resíduos de construção e de demolições, esse debate se torna ainda mais urgente. Desse modo, o objetivo geral deste artigo é tecer considerações sobre o $D f A D$ no contexto da Arquitetura Contemporânea Brasileira através da investigação de publicações recentes realizadas por pesquisadores nacionais. Para isso, realizou-se uma Revisão Sistemática da Literatura (RSL) em que observou-se uma baixa quantidade de produções na temática, além do grande foco nas tipologias habitacionais, principalmente as de interesse social (HIS). Portanto, diante do analisado, posiciona-se nesse artigo a necessidade de ampliação das discussões do $D f A D$ no cenário nacional, principalmente após os desafios impostos pelo contexto pandêmico.

Palavras-chave: design para adaptabilidade; flexibilidade; arquitetura brasileira; revisão sistemática da literatura.

\section{Introdução}

As consequências do aquecimento global têm se mostrado mais explicítas em diversas regiões ao redor do mundo. De acordo com o Intergovernmental Panel on Climate Change Report (IPCC, 2021), muitas dessas mudanças já podem ser consideradas irreversíveis. O recente Report trouxe reflexões sobre a influência humana no clima, enfatizando as consequências das crescentes emissões de $\mathrm{CO}^{2}$ na atmosfera, as maiores responsáveis pelas mudanças climáticas.

Tais questões se revelam ainda mais urgentes na Arquitetura, já que projetar sem considerar o futuro representa uma prática fundamentalmente insustentável. Os arquitetos devem ser cada vez mais estimulados a responder às necessidades humanas e aos fatores externos, não apenas atentando para as novas construções, mas também para as pré-existentes. Assim, considerações acerca do ciclo de vida são ainda mais demandadas. 
Diante desse contexto, é evocado neste artigo o papel do Design para Adaptabilidade (DfAD) nos debates sobre a Arquitetura atual. Schmidt III e Austin (2016, p.45) definem a Adaptabilidade como "a capacidade de um edifício em acomodar efetivamente as demandas em evolução de seu contexto, maximizando assim o seu valor ao longo da vida". Portanto, responder adequadamente à mudanças seria uma das principais características por trás do conceito (GOSLING et al 2013).

Essa temática vem ganhando cada vez mais relevância em pesquisas no mundo, considerando-a como a resposta central frente às problemáticas da obsolescência (KOUGEA, 2019) e da redundância (HEIDRICH et al., 2017). Em geral, edifícios adaptáveis podem ser utilizados por um período maior que os não (ROCKOW, ROSS, BLACK, 2018) e isso pode reduzir a quantidade de demolições tanto em tempos presentes quanto futuros (diminuindo consequentemente emissões de $\mathrm{CO}^{2}$ ).

De forma geográfica específica e considerando o cenário do Brasil em suas dimensões territoriais e populacionais expressivas (IBGE, 2021) - onde existem previsões no aumento da geração de resíduos sólidos urbanos (RSU) incluindo os de construções e demolições (ABRELPE, 2020)-, observou-se a necessidade de explorar o $D f A D$ de acordo com a perspectiva nacional. Logo, o objetivo geral deste artigo é tecer considerações sobre a temática do Design para Adaptabilidade (DfAD) na Arquitetura Contemporânea Brasileira com base na realização de uma Revisão Sistemática da Literatura (RSL) em publicações recentes realizadas por pesquisadores do país.

Essa investigação se enquadra como parte da investigação de uma dissertação em Design realizada de modo integrado à uma pesquisa em desenvolvimento pelo Adaptable Futures Group ${ }^{i}$, grupo de pesquisa que têm se debruçado desde 2020 no estudo do $D f A D$ na Arquitetura Contemporânea Brasileira.

\section{Metodologia}

Para atingir o objetivo geral dessa pesquisa, realizou-se uma Revisão Sistemática da Literatura (RSL), seguindo, por isso, critérios rigorosos. A RSL permite a identificação de lacunas e ainda serve como base para definição de pesquisas futuras (PAUL, CRIADO, 2020). Tranfield, Denyer e Smart (2003) definem um protocolo de desenvolvimento dessa revisão em 3 estágios, sendo elas o planejamento, a condução e a disseminação. Com esses em mente, definiu-se as fases da RSL, dentre as principais: (1) a identificação da necessidade da revisão, buscando entender a temática exposta no contexto recente brasileiro; (2) a seleção das publicações em bases de dados de escala internacional e nacional; (3) a organização e exclusão dos estudos, seja por área de estudo ou por repetição; (4) a síntese. 
Através de uma RSL realizada por Askar, Bragança e Gervásio (2021) com 181 artigos publicados desde 2015 na língua inglesa, ou seja, com um olhar mais ampliado sobre o que tem sido publicado recentemente, identificou-se três grandes eixos de abordagem nas publicações quanto ao conceito de Adaptabilidade, sendo eles: interpretação e caracterização do conceito; dimensões e conceitos sobrepostos (dentre eles o de Flexibilidade); promoção de tendências e facilitadores projetuais.

Assim, de forma específica, a investigação base desse artigo buscou responder à pergunta "quais os principais enfoques do $D f A D$ presentes na literatura dos últimos dez anos produzidas por pesquisadores nacionais?". A RSL focou, assim, em produções publicadas entre 2010 a 2020 nas bases de dados Scielo, Web of Science e Scopus (escala internacional) e teve a Biblioteca Digital Brasileira de Teses e Dissertações (BDTD) como principal base para teses e dissertações (escala nacional). A pesquisa foi realizada no mês de Junho de 2021. O Mendeley foi a principal ferramenta de organização durante as fases de coleta e posterior análise, conjuntamente com o Excel.

No geral, foram utilizados os termos "adaptabilidade" ou "flexibilidade" como fitros e o operador booleano $O R$, já que, em algumas pesquisas, ainda não há clareza dessas definições, utilizando tais conceitos ora como sinônimos ora como complementares. Nesse estudo, entende-se a Adaptabilidade como sendo o conceito mais amplo e Flexibilidade como parte dessa, então decidiu-se aqui extender as buscas para se obter um resultado mais abrangente. As publicações foram ainda limitadas à área temática da "Arquitetura" e, nas bases de dados internacionais, ao país "Brasil". Para uma coleta inicial ainda mais vasta, foi decidido não definir na busca o foco apenas no título, keywords e abstract.

234 publicações foram encontradas no total, mas apenas 15 foram consideradas relevantes ao estudo. Os critérios de exclusão foram: o não enquadramento ao escopo da pesquisa (muitas vezes por ser de outra área, como a de Computação e Engenharias); e os duplicados. Por fim, foram adicionadas ainda 16 publicações encontradas no Google Acadêmico, uma base que possui uma maior cobertura, totalizando 31 publicações (Figura 1).

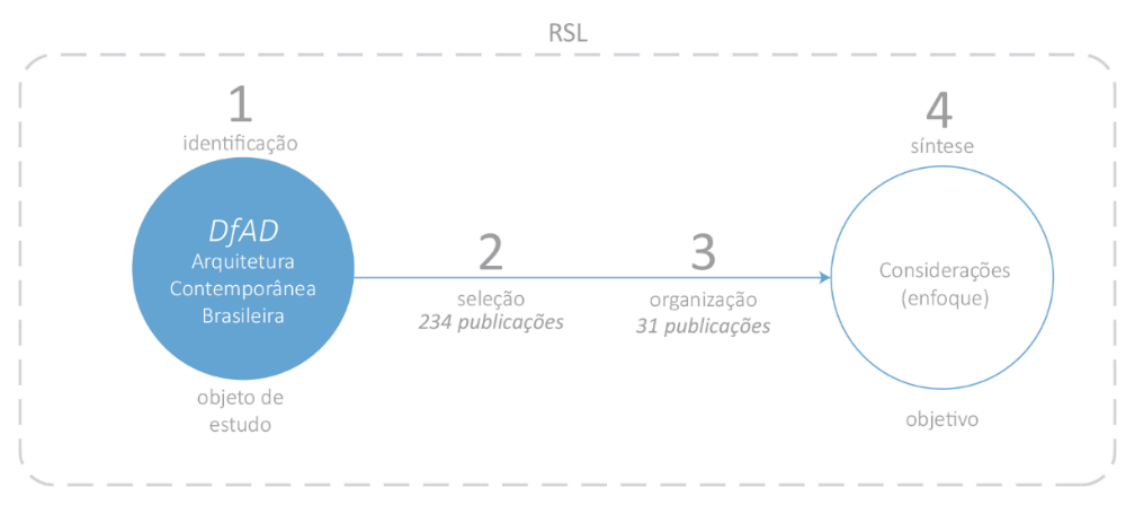

Figura 1. Metodologia e Objetivo.

Fonte: Feito pelos autores. 


\section{O Design para Adaptabilidade $(D f A D)$ e a Arquitetura Contemporânea Brasileira}

A utilização do termo Design para $X(D f X)$ no Design é adotado para caracterizar instrumentos que tem por objetivo a abordagem de temas específicos (a variante $X$ ), através da geração de diretrizes e métodos com foco no desenvolvimento de um produto. O Design para Adaptabilidade surgiu, assim, como uma forma de visualização da produção pelas demandas em mudança no mercado, tanto nas fases iniciais quanto nas finais de utilização de um artefato (GODIM, 2010).

Esse contexto trás à tona a percepção do ciclo de vida dos produtosii. As negativas consequências que surgem com o impacto das mudanças climáticas mundialmente tornam esse fator ainda mais importante a ser considerado no ato projetual. Em muitas áreas do Design, como por exemplo o Ecodesign, existe uma preocupação pela redução dos impactos ambientais considerando a vida útil dos produtos (SCHÄFER, LÖWER, 2021). Portanto, a noção de circularidade parece ser cada vez mais uma abordagem de articulação entre ambos designers e stakeholders:

....as fases de design e final de vida do ciclo de vida do produto devem ser conectadas para garantir a circularidade dos componentes e materiais. Portanto, os designers devem levar isso em consideração ao projetar para e a partir do final da vida útil. 0 corolário é que as partes interessadas no fim da vida útil do produto também devem mudar suas práticas para trabalhar a partir e para o design (LEAL et al, 2020).

De modo integrado à Arquitetura, o olhar para o $D f A D$ tem se caracterizado como um tópico de interesse mundial, além de uma resposta prioritária às questões climáticas. Segundo Askar, Bragança e Gervásio (2021, p.2), “o valor essencial da adaptabilidade surge - em grande parte - da impossibilidade de prever futuras mudanças". Eles pontuam ainda a importância sustentável do uso de estratégias projetuais adaptáveis na minimização do consumo de energia e materiais.

Essa pesquisa propõe que o Design para Adaptabilidade (DfAD) seja uma alternativa potencial para abarcar as complexas demandas do mundo contemporâneo, uma resposta às mudanças em fluxo. Sabe-se que o $D f A D$ proporciona diversos benefícios, dentre eles: ambientais, diminuindo a poluição ao meio ambiente; além de resilientes, com utilização de materiais simples, duráveis e de baixo custo de manutenção; comunitários, preservando o valor cultural e criando comunidades saudáveis; e econômicos, através do inerente maior valor implícito (MELTON, 2020). Nesse sentido, os arquitetos precisam ser verdadeiros exploradores do Design para Adaptabilidade (DfAD) em sua produção.

De forma mais pontual, têm-se como objeto de estudo o $D f A D$ na Arquitetura Contemporânea Brasileira, com base na justificativa de gerar estímulos para o protagonismo do Brasil nessa temática. Embora a presença da noção de Adaptabilidade na arquitetura nacional não seja recente, já que a 
adaptação ao meio e às condições locais, por exemplo, tenha sido uma busca dos períodos neocoloniais e modernistas (CORREIA, 2009), prevê-se que o maior país da América Latina em termos territoriais e populacionais demandará um grande número de construções no futuro, principalmente de moradias, em decorrência da alteração do perfil demográfico (CBCS, 2015) e isso afetará diretamente o setor da construção civil no país. Por tais motivos, o entendimento do $D f A D$ no contexto do Brasil, tem sido, desde 2020, objeto de investigação do Adaptable Futures Group, um grupo que investiga a Adaptabilidade no ambiente construído, e que é tomado aqui como principal referência (Figura 2).

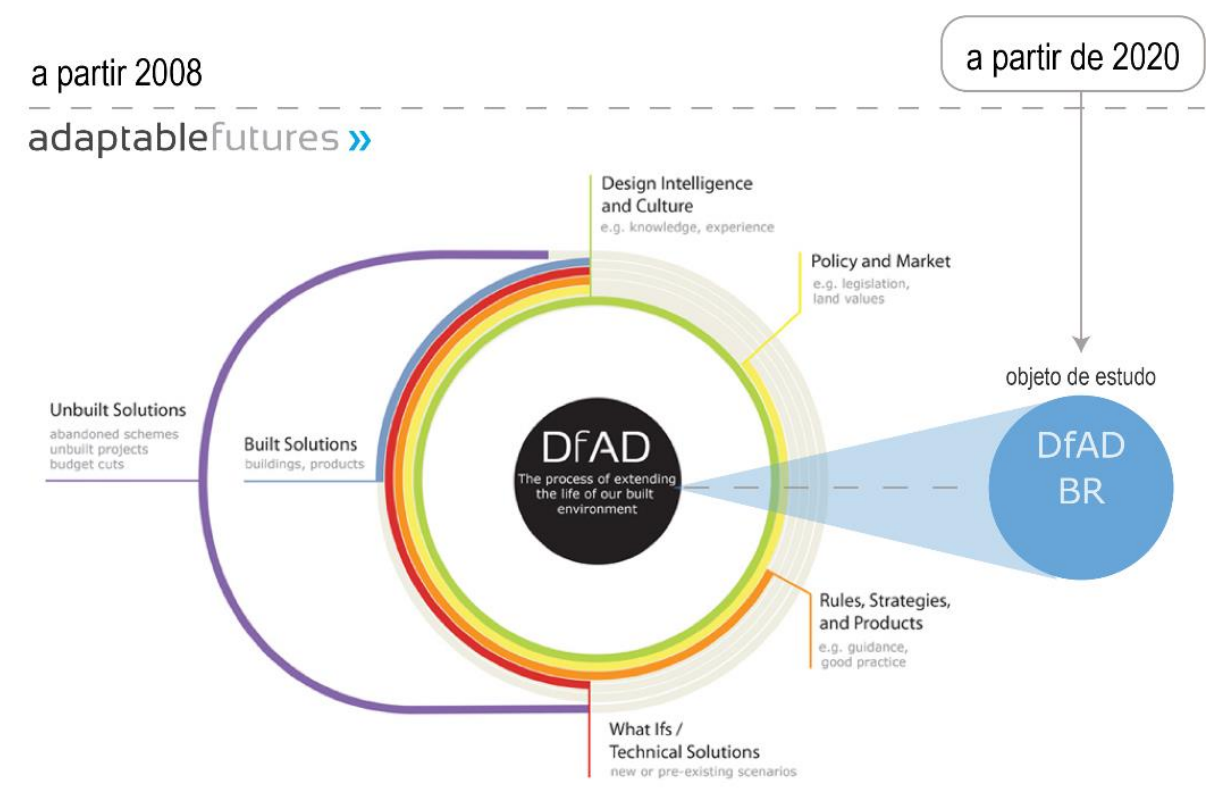

Figura 2. Objeto de estudo: $O$ DfAD na Arquitetura Contemporânea Brasileira.

Fonte: Feito pelos autores com base em SCHMIDT III, AUSTIN, 2016, p. 158.

Abordando o DfAD como um "processo para extender a vida do nosso ambiente construído" (SCHMIDT III, AUSTIN, 2016, p. 158), o livro Adaptable Architecture: Theory and Practice lança um manifesto para Adaptabilidade, no qual uma das proposições é "Adaptabilidade é específica ao contexto". Os autores afirmam que o entendimento da Adaptabilidade de forma universal não existe e cada projeto tem suas características peculiares. É diante dessa perspectiva que essa pesquisa incita a relevância das investigações do $D f A D$ no cenário brasileiro, de forma a auxiliar no estímulo a debates sobre o tema na Arquitetura Contemporânea à nível nacional. 


\section{Resultados e Discussões}

A realização da RSL possibilitou a geração de algumas considerações iniciais sobre o $D f A D$ no contexto da Arquitetura Contemporânea Brasileira. A primeira delas foi: (1) a baixa quantidade de publicações nacionais coletadas nas bases de dados exploradas, o que representa uma grande lacuna para pesquisas futuras. De acordo com a base BDTD, apenas 11 dissertações e 1 tese foram registradas na última década no tema (número ampliado para um total de 16 considerando-se as adições posteriores advindas do Google Acadêmico).

Além disso, notou-se que (2) grande parte do enfoque das mesmas dá-se na (i) tipologia habitacional (mais de $60 \%$ das publicações), principalmente nas habitações de interesse social (HIS), citando programas como o Minha Casa Minha Vida (MCMV). Essa constatação havia sido pontuada em uma RSL realizada em 2017 por Costa, Logsdon e Fabrício (2017), que, apesar de não ter a produção nacional como foco, justificam esse destaque pela política adotada no país nos últimos anos. Isso demonstra uma positiva tendência para análises futuras que podem ser ainda mais aprofundadas.

Outros enfoques observados foram: (ii) projeto/proposta, nas quais mais da metade são habitacionais; (iii) outras tipologias; e, em menor quantidade, foco em temas como (iv) pré-fabricação, teoria/história, conceituação do tema e publicações. Apesar de menores, esses números indicam alguns caminhos relevantes a serem ampliados para exploração da temática (Tabela 1).

Tabela 1. Enfoque das publicações recentes (últimos 10 anos) por pesquisadores brasileiros.

\begin{tabular}{|c|c|c|c|}
\hline $\begin{array}{l}\text { ENFOQUE } \\
\text { PRINCIPAL }\end{array}$ & $\begin{array}{l}\text { PUBLICAÇÕES } \\
\text { (ver referências) }\end{array}$ & QUANTIDADE & PORCENTAGEM \\
\hline (i) Habitação & $\begin{array}{l}(3),(6), 8),(15),(18), \\
(20),(23),(25),(30)\end{array}$ & 19 & $61,29 \%$ \\
\hline HIS & $\begin{array}{c}(1),(5),(9),(16),(19) \\
(21),(22),(26),(28)\end{array}$ & 10 & $32,25 \%$ \\
\hline (ii) Projeto/ Proposta & (7), (10) & 5 & $16,12 \%$ \\
\hline Habitação & $(12),(13),(29)$ & 3 & $9,67 \%$ \\
\hline $\begin{array}{l}\text { (iii) Outras tipologias } \\
\text { (ex: pavilhões, edifícios } \\
\text { públicos, escolar) }\end{array}$ & (2), (4), (14), (17) & 4 & $12,90 \%$ \\
\hline $\begin{array}{l}\text { (iv) Outros temas } \\
\text { (pré-fabricação, teoria/história, } \\
\text { conceituação, publicações) }\end{array}$ & (24), (27), (31) & 1 cada & $3,22 \%$ cada \\
\hline TOTAL & (1) a (31) & 31 & $100 \%$ \\
\hline
\end{tabular}


Apesar da RSL ter se concentrado em bases de dados específicas, ela foi importante para gerar o posicionamento exposto nesse artigo e base para o projeto de dissertação em desenvolvimento: o Brasil precisa aumentar e priorizar a produção no tema para ocupar um lugar de destaque nas contribuições sobre o $D f A D$ de forma global. Considerando a pouca produção coletada e sendo o maior enfoque delas em uma tipologia em específico (habitacional), percebe-se um grande espaço de abordagem para pesquisas futuras, incluindo não apenas as linhas aqui destacadas, mas também, explorando o $D f A D$ de forma mais ampla no contexto nacional.

\section{Conclusões}

A adequação às necessidades distintas é um dos fatores chave no Design para Adaptabilidade (DfAD), principalmente na Arquitetura, quando considera-se que a não-resposta às mudanças pode acarretar na obsolescência e futura demolição de edificações. Assim, faz-se fundamental investigar a problemática do ciclo de vida, agregada às discussões sobre as mudanças climáticas, emissões de $\mathrm{CO}^{2}$ e ao contexto pandêmico mais recente ocasionado pelo covid-19, de modo a ampliar ainda mais os debates sobre o $D f A D$ e suas estratégias de implementação.

No Brasil, em específico, pressupõe-se que o $D f A D$ tenha no país um cenário expressivo de produção e discussões que extrapolem as particularidades das tipologias habitacionais que foram destacadas na RSL como sendo o enfoque principal de publicações nacionais na última década. Portanto, o maior desafio baseia-se, essencialmente, no aumento e consolidação dos debates em ambas Academia e prática profissional.

\section{Referências}

ABRELPE. Panorama dos resíduos sólidos no Brasil 2020. 2020.

ASKAR, Rand; BRAGANÇA, Luís; GERVÁSIO, Helena. Adaptability of buildings: a critical review on the concept evolution. Applied Sciences, v. 11, n. 10, 2021.

CBS, Conselho Brasileiro de Construção Sustentável. Aspects of sustainable construction in Brazil and public policy promotion: subsidies for promoting sustainable construction. Versão 1, 2015.

CORREIA, Telma de Barros. Arquitetura e Ambiente: a noção de adaptabilidade ao meio no discurso modernista. Pós, v.16, n.25, 2009.

COSTA, Heliara Aparecida; LOGSDON, Louise; FABRíCIO, Márcio Minto. Flexibilidade em projetos de arquitetura: contribuições a partir de uma revisão sistemática da literatura. PARC Pesquisa em Arquitetura e Construção, Campinas, v. 8, n. 3, p. 144-160, 2017. 
GODIM, Cristina. Critérios para seleção de conexões em Mobiliário orientado para Adaptabilidade. 2010. 193 f. Dissertação (Mestrado em Design) - Pós Graduação em Design, Universidade Federal do Rio Grande do Sul, Porto Alegre, 2010.

GOSLING, Jonathan, et al. Adaptable buildings: A systems approach. Sustainable Cities and Society, v. 7, p.44-51, 2013.

HEIDRICH, Oliver, et al. A critical review of the developments in building adaptability. International Journal of Building Pathology and Adaptation, v. 35, n. 4, p. 284-303, 2017.

IBGE PAÍSES. Brazilian Institute of Geography and Statistics. Disponível em: https://paises.ibge.gov.br Acesso em: 11 de jul. de 2021.

IPCC, Intergovernmental Panel on Climate Change. IPCC Press Release: Climate change widespread, rapid, and intensifying. Disponível em: https://www.ipcc.ch/2021/08/09/ar6-wg1-20210809-pr/ Acesso em: 12 de ago. de 2021.

KOUGEA, Charitini. Degrees of Adaptability: A design Framework for Adaptable Real Estate Transformation Projects. Master Thesis: TU Delft, 2019.

LEAL, Jorge Martinez. et al. Design for and from recycling: a circular ecodesign approach to improve the circular economy. Sustainability, v.12, n.23, 2020.

MELTON, Paula. Buildings that last: Design for Adaptability, Deconstruction and Reuse. The American Institute of Architects, 2020.

PAUL, Justin; CRIADO, Alex Rialp. The art of writing literature review: what do we know and what do we need to know? International Business Review, v. 29, n. 4, 2020.

SCHÄFER, Malte; LÖWER, Manuel. Ecodesign: a review of reviews. Sustainability, v.13, n.1, 2021.

SCHMIDT III, Robert; AUSTIN, Simon. Adaptable Architecture: Theory and Practice. Abingdon: Routledge, 2016.

ROCKOW, Zoraya Roldán; ROSS, Brandon; BLACK, Anna K. Review of methods for evaluating adaptability of buildings. International Journal of Building Pathology and Adaptation, v. 37, n. 3, p.273-287, 2018.

TRANFIELD, David; DENYER, David; SMART, Palminder. Towards a methodology for Developing Evidence-Informed Management Knowledge by Means of Systematic Review. British Journal of Management, v. 14, p. 207-222, 2003.

\section{Publicações selecionadas pela RSL}

1. BRANDÃO, Douglas Queiroz. Disposições técnicas e diretrizes para projetos de habitação sociais evolutivas. Ambiente Construído, v. 11, n. 2, p.73-96, 2011.

2. FONTES, Adriana Sansão; GALARCE, Fernando Espósito. A condição efêmera na cidade contemporânea: três pavilhões temporários no Rio de Janeiro (2012-2018). Arquitecturas del Sur, v. 38, n. 57, p.20-37, 2020. 
3. PAULICHEN, Luana; LEITE, Raquel M.; PINA; Silvia A. Mikami G. Resilience in Architecture: Housing as a process. Strategic Design Research Journal, v. 12, n. 2, p.383-401, 2019.

4. SALVIONI, Amanda Malchiaffava. Implantação de edifícios padrão para equipamentos públicos comunitários em diferentes terrenos: recomendações para flexibilidade na concepção de novos projetos. 2015. 223 f. Dissertação (Mestrado em Arquitetura e Urbanismo) - Pós Graduação em Arquitetura e Urbanismo, Universidade Estadual de Londrina, Londrina, 2015.

5. LOGSDON, Louise. O Programa Minha Casa, Minha Vida em Cuiabá-MT: uma análise da qualidade dos projetos destinados às famílias de baixa renda. 2012. $183 \mathrm{f}$. Dissertação (Mestrado em Arquitetura e Urbanismo) - Pós Graduação em Arquitetura e Urbanismo, Universidade Federal de Santa Catarina, Florianópolis, 2012.

6. WEINSCHENCK, Julia Hunsche. Estudo da flexibilidade como mecanismo para personalização de casas pré-fabricadas: uma abordagem voltada para industrialização de casas de madeira. 2012. 146 f. Dissertação (Mestrado em Arquitetura e Urbanismo) - Pós Graduação em Arquitetura e Urbanismo, Universidade Federal de Santa Catarina, Florianópolis, 2012.

7. QUEIROZ, Marise Brito Silva Câmara de. Ampliação do setor de aulas teóricas IV UFRN: proposta arquitetônica baseada no conceito da flexibilidade e adaptabilidade, utilizando os parâmetros do conforto ambiental. 2014. 126 f. Dissertação (Mestrado Profissional em Projeto e Meio Ambiente) - Pós Graduação de Arquitetura, Projeto e Meio Ambiente, Universidade Federal do Rio Grande do Norte, Natal, 2014.

8. BARBOSA, Mirella de Souza. Arquitetura flexível: um desafio para uma melhor qualidade habitacional. 2016. 133 f. Dissertação (Mestrado em Arquitetura e Urbanismo) - Pós Graduação em Arquitetura e Urbanismo, Universidade Federal da Paraíba, João Pessoa, 2016.

9. LEMOS, Nayara dos Santos. Habitações flexíveis e adaptáveis: o estudo da aplicabilidade de soluções espaciais do Solar Decathlon Europe para o conjunto Habitacional Gervásio Maia de Pessoa-PB, Brasil. 2015. 154 f. Dissertação (Mestrado em Arquitetura e Urbanismo) - Pós Graduação em Arquitetura e Urbanismo, Universidade Federal da Paraíba, João Pessoa, 2015.

10. BARROS, Dominique. Campus 5 - uma proposta de residência universitária interativa. 2017. 174 f. Dissertação (Mestrado Profissional em Projeto e Meio Ambiente) - Pós Graduação de Arquitetura, Projeto e Meio Ambiente, Universidade Federal do Rio Grande do Norte, Natal, 2017.

11. LEGONDE, Claúdia Kraemer. Flexibilidade em edificações residenciais: diretrizes de projeto e análise da aplicação no mercado imobiliário brasileiro. 2017. 207 f. Dissertação (Mestrado em Arquitetura e Urbanismo) - Pós Graduação em Arquitetura e Urbanismo, Projeto e Meio Ambiente, Universidade Vale do Rio dos Sinos, São Leopoldo, 2017.

12. MACEDO, Lílian Brito de. Como uma onda no mar: projeto arquitetônico de uma habitação evolutiva com princípios de racionalização. 2018. 164 f. Dissertação (Mestrado Profissional em Projeto e Meio Ambiente) - Pós Graduação de Arquitetura, Projeto e Meio Ambiente, Universidade Federal do Rio Grande do Norte, Natal, 2018.

13. RIBEIRO, Sandra Albino. Esquina Edifício: uma proposta arquitetônica para um edifício misto. 2014. 133 f. Dissertação (Mestrado Profissional em Projeto e Meio Ambiente) - Pós Graduação 
de Arquitetura, Projeto e Meio Ambiente, Universidade Federal do Rio Grande do Norte, Natal, 2014.

14. TALIN, Layla Christine Alves. Inter-relações entre aspectos arquitetônicos e a acústica em espaços adaptados para prática musical. 2013. 117 f. Dissertação (Mestrado em Arquitetura e Urbanismo) - Pós Graduação em Arquitetura e Urbanismo, Projeto e Meio Ambiente, Universidade Federal de Viçosa, Viçosa, 2013.

15. JORGE, Liziane de Oliveira. Estratégias de flexibilidade na arquitetura residencial multifamiliar. 2012. $511 \mathrm{f}$. Tese (Doutorado em Arquitetura e Urbanismo), Universidade de São Paulo, São Paulo, 2012.

16. VILLA, Simone Barbosa; STEFANI, Ana Carolina de Oliveira; OLIVEIRA, Natália Fleury Guedes de. Análise da flexibilidade no conjunto habitacional de interesse social do Programa "Minha Casa Minha Vida": o caso do loteamento Sucesso Brasil, Uberlândia. In: Simpósio Brasileiro de Qualidade do Projeto no Ambiente Construído, p. 892-905, 2019.

17. ALBERTO, Klaus Chave; SINDER, Marcela Barros. A flexibilidade no espaço escolar: variações sobre a compreensão do tema e da prática projetual. In: V PROJETAR, 2011, Belo Horizonte. V PROJETAR - Processos de projeto: teorias e práticas, p. 01-17, 2011.

18. SCHMITZ, Lisana; KUCHPIL, Eneida; PACHECO, Paulo C. B. Habitar coletivo: uma experiência no atelier de projeto. NPGAU/UFMG, 2011.

19. XAVIER, Regina do Nascimento Gomes; BARBIRATO, Gianna Melo. Análise quanto à flexibilidade espacial de unidades residenciais de conjunto habitacional em Maceió/AL. In: 20 Simpósio Brasileiro de Qualidade do Projeto no Ambiente Construído, p. 401-411, 2011.

20. VASCONCELOS, Claudia; BARTH, Fernando. Avaliação da Flexibilidade Arquitetônica em Habitações com dimensões reduzidas em Florianópolis. Revista Território, Espaço Construído e Meio Ambiente, v. 1, n.1, 2015.

21. PETENO, Elvis Alexandre; CAPELIN, Luana Jéssica; TRENTINI, Larissa Dias. A importância das disposições técnicas e diretrizes para projetos de habitações de interesse social (HIS) saudáveis. Akrópolis, v. 28, n.2, p. 233-242, 2020.

22. MOREIRA, Fernando DINIZ; HENRIQUES, José Evandro de Moura Rosa. Adaptabilidade na Arquitetura: um protótipo para comunidades carentes no Recife. Revista Projetar: Projeto e Percepção do Ambiente, v. 4, n.1, p. 126-140, 2019.

23. PARREIRA, Fernanda Vilela Martins; VILLA, Simone Barbosa. Resiliência na Habitação Social: avaliação dos impactos relacionados a sua (in)flexibilidade. VI ENANPARQ, 2020.

24. BRAGA, Bruno Melo. A flexibilidade como atributo da Arquitetura Moderna Brasileira e sua vigência na contemporaneidade. V Seminário Docomomo, 2016.

25. FREITAS, Rodrigo Rocha de. Flexibilidade e Adaptabilidade como estratégias projetuais: análise de edifícios de apartamentos contemporâneos em Belo Horizonte. 2020. 151 f. Dissertação (Mestrado em Arquitetura e Urbanismo), Universidade Federal de Minas Gerais, Belo Horizonte, 2020. 
26. BORTOLI, Karen Carrer Ruman de; VILLA, Simone Barbosa. Adequação ambiental como atributo facilitar da resiliência no ambiente construído em Habitações de Interesse Social. Ambiente Construído, v.20, n. 1, p. 391-422, 2020.

27. PIRES, Nayara. As manifestações da flexibilidade na Arquitetura. 2018. 176 f. Dissertação (Mestrado em Arquitetura e Urbanismo), Universidade Presbiteriana Mackenzie, São Paulo, 2018.

28. PARREIRA, Fernanda Vilela Martins. Estratégias de Flexibilidade orientadas ao usuário como facilitador da resiliência em habitação de interesse social. 2020. 297 f. Dissertação (Mestrado em Arquitetura e Urbanismo), Universidade Federal de Uberlândia, Uberlândia, 2020.

29. JÚNIOR, Francisco da Rocha Bezerra. Habitação Social Evolutiva: estratégias de flexibilidade para elaboração de projetos de habitação de interesse social. Uma proposta para Mãe Luzia/ Natal-RN. 2016. 255 f. Dissertação (Mestrado Profissional em Projeto e Meio Ambiente) - Pós Graduação de Arquitetura, Projeto e Meio Ambiente, Universidade Federal do Rio Grande do Norte, Natal, 2016.

30. MENDONÇA, Rafaela Nunes; VILLA, Simone Barbosa. Racionalizar e flexibilizar: um caminho para uma moradia participativa. III ENANPARQ, São Paulo, 2014.

31. COSTA, Heliara Aparecida; LOGSDON, Louise; FABRÍCIO, Márcio Minto. Flexibilidade em projetos de arquitetura: contribuições a partir de uma revisão sistemática da literatura. PARC Pesquisa em Arquitetura e Construção, Campinas, v. 8, n. 3, p. 144-160, 2017.

\footnotetext{
'O Adaptable Futures Group (AF) é um grupo de pesquisa internacional baseado na Loughborough University, no Reino Unido, que foca no projeto, construção e gerenciamento de um ambiente construído mais adaptável. Desde 2008, o Grupo investiga a Adaptabilidade em detalhes, olhando para a complexa teia de dependências que induzem, atrapalham e acomodam mudanças.

ii Entende-se nessa pesquisa os edifícios como produtos da Arquitetura.
} 